\title{
The New Ophthalmology Internship: A Trainee Curricular Survey
}

\author{
Tavish Nanda, MD ${ }^{1}$ Dan Gong, MD $\quad$ Royce W. S. Chen, MD ${ }^{1}$ George A. Cioffi, MD \\ Gustavo De Moraes, $\mathrm{MD}^{1}$ Lora R. Dagi Glass, MD ${ }^{1}$ \\ ${ }^{1}$ Edward S. Harkness Eye Institute, Columbia University Irving Medical \\ Center, New York, New York \\ 2 Massachusetts Eye and Ear Infirmary, Harvard Medical School, \\ Boston, Massachusetts

\begin{abstract}
Address for correspondence Tavish Nanda, MD, Edward S. Harkness Eye Institute, 635 W 165th Street, First Floor, New York, NY 10032 (e-
\end{abstract} \\ mail: tn2290@cumc.columbia.edu).
}

J Acad Ophthalmol 2020;12:e134-e142.

\begin{abstract}
Keywords

- curriculum

- internship

- Accreditation Council of Graduate Medical Education

- survey

- residency

- ophthalmology training

- interdisciplinary

- extramural

Background The American Academy of Ophthalmology, in conjunction with the Accreditation Council of Graduate Medical Education (ACGME), has mandated the creation of a linked ophthalmology internship year for all programs by 2021. This mandate provides a unique opportunity to tailor a curriculum specific to the ophthalmology trainee. This study reports the results of a national survey of ophthalmology residents and fellows regarding the relevance and applicability of extramural content to their ophthalmology training. Methods Using the online portal SurveyMonkey, an anonymous survey was sent to 119 ACGME-accredited ophthalmology residencies. It consisted of nine questions formulated to measure the perceived applicability and relevance of other specialties to ophthalmology training. Responses were summarized with descriptive statistics. Outcomes were compared by one-way analysis of variance with Tukey's post hoc analysis for between group differences and Benjamini-Hochberg for multiple comparisons.

Results Ninety-four respondents (residents and fellows) completed the survey (January-February 2020); 52.1\% completed an internal medicine internship, 42.6\% transitional, and $5.3 \%$ general surgery. Overall, prior internship experience was rated favorably. Otolaryngology and radiology were deemed the most relevant to ophthalmology training. Intensive care unit (ICU) was considered the least beneficial, followed by general surgery, primary care, and pediatrics. Neuroradiology was overwhelmingly preferred over general radiology. The majority of respondents reported minimal exposure to oral and maxillofacial surgery (OMFS) in medical school/internship. When choosing a reason for inclusion in the new curriculum, respondents reported a high level of surgical overlap with otolaryngology, OMFS, and plastic surgery; clinical overlap with neurology; and ophthalmic consultation with emergency medicine.

Conclusion Several specialties-notably otolaryngology, radiology, and neurology-were considered highly relevant but lacking in preresidency exposure. The preference for neuroradiology was almost unanimous. In contrast, general surgery and ICU were found to be minimally relevant. Overall, ophthalmology residents reported a high level of satisfaction with their internship experience, an important consideration when building a curriculum that will appeal to potential applicants. The findings of this survey provide a framework to build a curricular plan based on current resident and fellow experience.
\end{abstract}

received

April 26, 2020

accepted after revision

July 17,2020
DOI https://doi.org/

10.1055/s-0040-1716363. ISSN $2475-4757$.
Copyright $\odot 2020$ by Thieme Medical

Publishers, Inc., 333 Seventh Avenue, New York, NY 10001, USA. Tel: +1(212) 760-0888.
License terms

(ब) (1) $\circledast$ 
In 2016, members of the Association of University Professors in Ophthalmology published a white paper highlighting the need to consider restructuring the postgraduate year (PGY) 1 internship year to allow for an expanded interdisciplinary curriculum and to incorporate further opportunities for ophthalmic education. ${ }^{1}$ Other specialties have made similar modifications, such as orthopaedics in 2013, otolaryngology (ears, nose, and throat [ENT]) in 2016, and urology in 2019, to focus intern year training towards their respective specialty needs. In 2003, the residents and fellows' section of the American Academy of Otolaryngology - Head and Neck Surgery, for example, found that more than $50 \%$ of incoming ENT residents missed rotations in anesthesia and emergency medicine during their intern year. This led to a new program mandate to incorporate internship into a 5-year residency. ${ }^{2}$ In particular, ENT programs reduced time in general surgery, replacing it with more diverse experiences such as critical care medicine, plastic surgery, laryngology, and head and neck oncology. ${ }^{3}$ Following suit, in 2019, the American Academy of Ophthalmology in conjunction with the Accreditation Council of Graduate Medical Education (ACGME) mandated the creation of a linked ophthalmology internship year for all ophthalmology programs by 2021 . This internship experience will include 3 months of ophthalmology but is otherwise open to a variety of curricular rotations. This new mandate will provide the opportunity to tailor intern year curriculum to meet the needs of the new-age ophthalmology trainee. Additionally, this mandate creates a unique opportunity to involve current trainees in discussions regarding curricular content. This study reports on results of a national survey of ophthalmology residents and fellows regarding the perceived importance and relevance of a variety of extramural rotations to their ophthalmology training.

\section{Methods}

This study was approved by the Institutional Review Board at Columbia University Irving Medical Center using the online portal SurveyMonkey. An anonymous survey was sent to the program coordinators of 119 ACGME-accredited programs across the United States; each coordinator was asked to forward the survey to their current trainees, including all current residents and fellows. It consisted of nine questions (-Fig. 1) formulated to measure the perceived applicability and relevance of other specialties to ophthalmology training. In addition, the survey measured whether or not a respondent had direct experience in each specialty and, if applicable, in what context (medical school, intern year, or both). For the purposes of anonymity, the authors did not solicit information on trainee's geographic location, age, gender, or race. Respondents were asked to rank program size based on previously published terminology regarding "small," "medium," and "large" programs, with small programs having $<4$ residents per year, medium 4 to 6 residents per year, and large $>6$ residents per year. ${ }^{4}$ Respondents were also asked whether they considered their program to be "urban" versus "nonurban," defined subjectively, to determine if training environment may play a role in specialty perception. The survey link remained open from January to February 2020.
Survey responses and participant characteristics were summarized with descriptive statistics. Outcomes between groups were compared using two sample $t$-test or one-way analysis of variance (ANOVA), with Tukey's honestly significant difference post hoc analysis for intergroup comparison, when applicable. The analysis was corrected for multiple comparisons by the Benjamini-Hochberg's method, to adjust $p$-values for both Type I and Type II errors. Subgroup analysis included prior extramural experience, postgraduate education level at the time of survey completion (PGYs 2-4), urban versus nonurban residency setting, and internship experience. To account for all variables collectively, a mixed model analysis was performed to determine if certain fixed factors (year of training, program size, etc.) remained significant when accounting for interrater correlation, in order to normalize the data to each respondent's baseline. Emergency medicine, nonurban, large $>6$ residents, general surgery internship, and internship rating $\geq 4 / 5$ (as a covariate) were used as reference groups.

\section{Results}

\section{Demographics}

Ninety-four residents and fellows chose to respond to the survey between January and February 2020. The total number of potential subjects who received a request to complete the survey is unknown since we relied on the impetus of each program to forward the survey to their trainees $(\sim 1,400$ total residents across 119 programs). ${ }^{5}$ Among the 94 who did respond, the survey took an average of 5 minutes and 13 seconds to complete. Eleven respondents were from small programs (11.7\%), and 12 respondents were from large programs $(12.7 \%)$. The majority of survey respondents (56.4\%) were from medium-sized urban programs, followed by medium-sized nonurban programs (18.1\%). A total of $35.1 \%$ were first-year ophthalmology residents (PGY 2), 29.8\% were PGY 3, 25.5\% were PGY 4, and 9.6\% were fellows. Most residents completed an internal medicine internship year $(52.1 \%)$ or transitional internship year (42.6\%), few completed general surgery internship (5.3\%), and none completed a pediatrics internship.

\section{Extramural Specialty Applicability}

Respondents were asked to rank a series of specialties on a scale of 1 to 10 , with 1 delineating a minimally relevant specialty for ophthalmology training and 10 delineating an extremely relevant specialty experience. ENT and radiology had the highest percentage of "extremely relevant" 9 to 10 rankings at 21.2 and $16.5 \%$, respectively. Intensive care unit (ICU) was considered the least beneficial, with an average rating of 2.80 , with $31.9 \%$ of respondents picking "minimally relevant." This was followed by general surgery (average 3.46, $19.2 \%$ minimally relevant), primary care (average $3.56,15.4 \%$ minimally relevant), and pediatrics (average 4.08, 9.9\% minimally relevant). The mean, standard deviation, median, and interquartile range for each specialty rating are reported in - Table 1, from highest to lowest.

Respondents were then asked to select up to three predetermined reasons as to why a specialty might be particularly 
1. What best describes your ophthalmology residency? ${ }^{*}$

$$
\begin{aligned}
& <4 \text { residents per year, non-urban } \\
& <4 \text { residents per year, urban } \\
& 4-6 \text { residents per year, non-urban } \\
& 4-6 \text { residents per year, urban } \\
& >6 \text { residents per year, non-urban } \\
& >6 \text { residents per year, urban }
\end{aligned}
$$

*Fellows were asked to respond according to their prior residency.

2. What year in training are you?

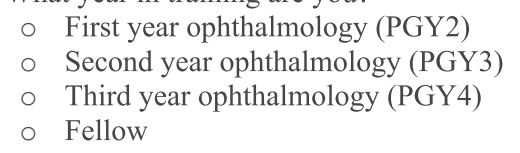

3. What intern year did you do?

$$
\begin{array}{ll}
\circ & \text { General surgery } \\
\circ & \text { Internal medicine } \\
\circ & \text { Transitional } \\
\circ & \text { Pediatrics }
\end{array}
$$

4. How would you rate your intern year experience (5-star scale)

5. Please answer the following questions for each specialty (17 specialties):

Please rate the specialty's applicability to ophthalmology:

- 1 (minimally relevant) to 10 (extremely relevant)

Did you have direct experience as a medical student or intern?

$$
\begin{array}{ll}
\circ & \text { Yes, medical school } \\
\circ & \text { Yes, intern year } \\
\circ & \text { Yes, both } \\
\circ & \text { No }
\end{array}
$$

Otolaryngology (ENT)

General Surgery

Plastic Surgery

Neurosurgery

Oral and Maxillofacial Surgery (OMFS)

Neurology (inpatient)

Neurology (outpatient)

Pediatrics

Radiology

\author{
Rheumatology \\ Endocrinology \\ Dermatology \\ Internal Medicine (inpatient) \\ Primary Care (outpatient) \\ Pre-operative Clearance \\ $\mathrm{ICU}$ \\ Emergency Medicine
}

6. Do you think neuro-ICU is more relevant than general ICU experience for ophthalmology residency?

$$
\begin{array}{ll}
\circ & \text { Yes } \\
\circ & \text { No } \\
\circ & \text { No difference }
\end{array}
$$

7. Do you think neuro-radiology is more relevant than general radiology experience for ophthalmology residency?

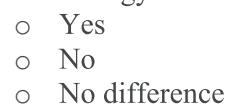

8. Please select the most relevant reasons for how each specialty may apply to ophthalmology (up to a MAX 3 per specialty). *The same 17 specialties as listed above were measured.*

- Patient referrals

- Clinical overlap

- Surgical overlap

- Ophthalmic consults

- Professional network

- Hospital logistics

- Diagnostic testing

- Not relevant

9. Any additional comments on the above questions/specialties welcome! $\circ$ Free Text Box

Fig. 1 Survey questions. Questions were built into the SurveyMonkey online portal. Both parts of question 5 were asked for each specialty listed, resulting in 17 rows of data collection. In question 8, respondents could choose up to three choices from the list provided for each specialty, resulting, again, in 17 rows of data collection. 
Table 1 Extramural specialty applicability to ophthalmology trainees (out of 10)

\begin{tabular}{|l|l|l|l|l|}
\hline Specialty & Mean & SD & Median & IQR \\
\hline ENT & 7.62 & 1.82 & 8.00 & 2.0 \\
\hline Radiology & 6.97 & 1.89 & 7.00 & 2.5 \\
\hline Neurology (inpatient) & 6.50 & 1.88 & 7.00 & 2.0 \\
\hline Plastic surgery & 6.39 & 1.80 & 7.00 & 1.0 \\
\hline Rheumatology & 6.24 & 1.76 & 6.00 & 3.0 \\
\hline OMFS & 5.90 & 2.12 & 6.00 & 2.0 \\
\hline Preoperative clearance & 5.83 & 2.63 & 6.00 & 4.0 \\
\hline Neurosurgery & 5.68 & 2.17 & 6.00 & 3.0 \\
\hline Emergency Medicine & 5.60 & 2.08 & 6.00 & 3.0 \\
\hline Neurology (outpatient) & 5.22 & 2.27 & 5.00 & 3.0 \\
\hline Dermatology & 5.09 & 2.10 & 5.00 & 2.0 \\
\hline Endocrinology & 4.70 & 2.08 & 5.00 & 3.0 \\
\hline $\begin{array}{l}\text { Internal medicine } \\
\text { (inpatient) }\end{array}$ & 4.64 & 2.11 & 5.00 & 3.0 \\
\hline Pediatrics & 4.07 & 1.98 & 4.00 & 2.0 \\
\hline $\begin{array}{l}\text { Primary care } \\
\text { (outpatient) }\end{array}$ & 3.56 & 1.84 & 3.00 & 3.0 \\
\hline General surgery & 3.46 & 1.83 & 3.00 & 3.0 \\
\hline ICU & 2.82 & 1.79 & 2.00 & 3.0 \\
\hline
\end{tabular}

Abbreviations: ENT, ears, nose, and throat; ICU, intensive care unit; IQR, interquartile range; OMFS, oral and maxillofacial surgery; SD, standard deviation.

Note: The mean, SD, median, and IQR for each specialty rating is reported from highest to lowest.

applicable to ophthalmology training (-Fig. 2). The top four reasons by percentage for each specialty are depicted in -Fig. 3. ENT (87.1\%), oral and maxillofacial surgery (OMFS) (78.9\%), and plastic surgery (91.2\%) were specialties considered to have high levels of surgical overlap, while dermatology (25.6\%) and general surgery (26.1\%) were not. Emergency medicine was the highest in ophthalmic consultation at $91.0 \%$. Neurology was notably relevant with $91.3 \%$ choosing clinical overlap for this specialty, compared with rheumatology (85.56\%), endocrinology (64.04\%), and dermatology (74.44\%). Primary care was the highest patient referral specialty at $61.1 \%$. While 35.9 and $23.3 \%$ of respondents felt general surgery and ICU were "not relevant" in any way, $0 \%$ of respondents felt similarly with regard to ENT and radiology. Radiology (83.2\%), followed by neurology (34.8\%) and rheumatology (20.0\%), were considered relevant for diagnostic testing. When forced to choose between neuro-ICU versus general ICU, $47.9 \%$ of respondents were in favor of neuro-ICU, $10.6 \%$ favored general ICU, and $41.5 \%$ chose "no difference." Neuroradiology was overwhelmingly preferred (95.7\%) over general radiology

\section{Direct Experience/Exposure}

After rating the specialties' applicability to ophthalmology, participants were asked to state whether they had direct experience in the same specialty in medical school and/or internship. When analyzing differences in overall specialty experience, there was no significant difference between urban versus nonurban residency programs, PGY level, and type of internship completed. Respondents had the least exposure to OMFS, with $88.6 \%$ having no exposure whatsoever, followed by preoperative clearance (64.3\%), dermatology (58.6\%), and neurosurgery (58.6\%) ( - Fig. 3). Respondent exposure to general surgery and pediatrics was more weighted toward medical school than internship. Respondent exposure to internal medicine, ICU, radiology, and emergency medicine, in contrast, was seemingly robust during internship.

Only $34.3 \%$ of respondents noted any inpatient neurology experience during internship, even though $94.7 \%$ completed an internal medicine or transitional year, and inpatient neurology was considered important (6.50/10). Respondents reported a range of exposure in ENT, which ranked the highest of all specialties (7.62/10): 40\% had no experience and $42.9 \%$ had exposure in medical school alone. Low levels of exposure were listed for plastic surgery, rheumatology, endocrinology, and dermatology as well.

\section{Subgroup Analysis}

Overall, respondents rated their own internship experience favorably, with an average of 4.03 on a five-point scale; $45.7 \%$ of respondents gave their intern year experience the highest rating. When divided by internship year type, general surgery had an average rating of 2.3 (5 respondents), internal medicine 3.9 (49), and transitional 4.4 (40).

On adjusted one-way ANOVA, respondents who chose "urban" to describe their residency location rated all specialties significantly higher than their nonurban counterparts, except for general surgery, neurosurgery, ENT, endocrinology, dermatology, and preoperative clearance (-Table 2). As a total average across all specialties, fellows gave a higher average rating than their resident counterparts $(p=0.004)$, giving an average of $5.73 / 10$, followed by PGY 4 (5.50/10), PGY 3 (5.15/10), and PGY 2 (5.10/10). When analyzed by specific specialty, only ENT appeared to be more highly valued by PGY 4 residents $(p=0.04)$, but this was not found to be statistically significant on post hoc analysis. There were also significant differences in overall specialty ratings by program size, with an average of $5.58 / 10$ for respondents from large programs, $5.28 / 10$ for medium, and $4.89 / 10$ for small ( $p=0.015)$. Tukey's post hoc analysis affirmed a statistically significant difference between large and small programs $(p<0.05)$, though not between large- and medium-sized programs.

To determine how intern year experience may have impacted the assessment of various specialties, these ratings were divided into three ratings groups: $\leq 2$ (12), 3 (13), or $\geq 4$ (69). Total $33.3 \%$ of respondents in the $\leq 2$ category were general surgery interns (this comprised $80 \%$ of the general surgery respondents) and $71.3 \%$ of all respondents rated their experience $\geq 4$; of these respondents, $50.75 \%$ completed a transitional year, $47.8 \%$ an internal medicine year, and $1.49 \%$ a general surgery year. When examining average specialty pertinence rankings, internship experience was not a significant determinant: internship rating $\leq 2$ corresponded to an average of 


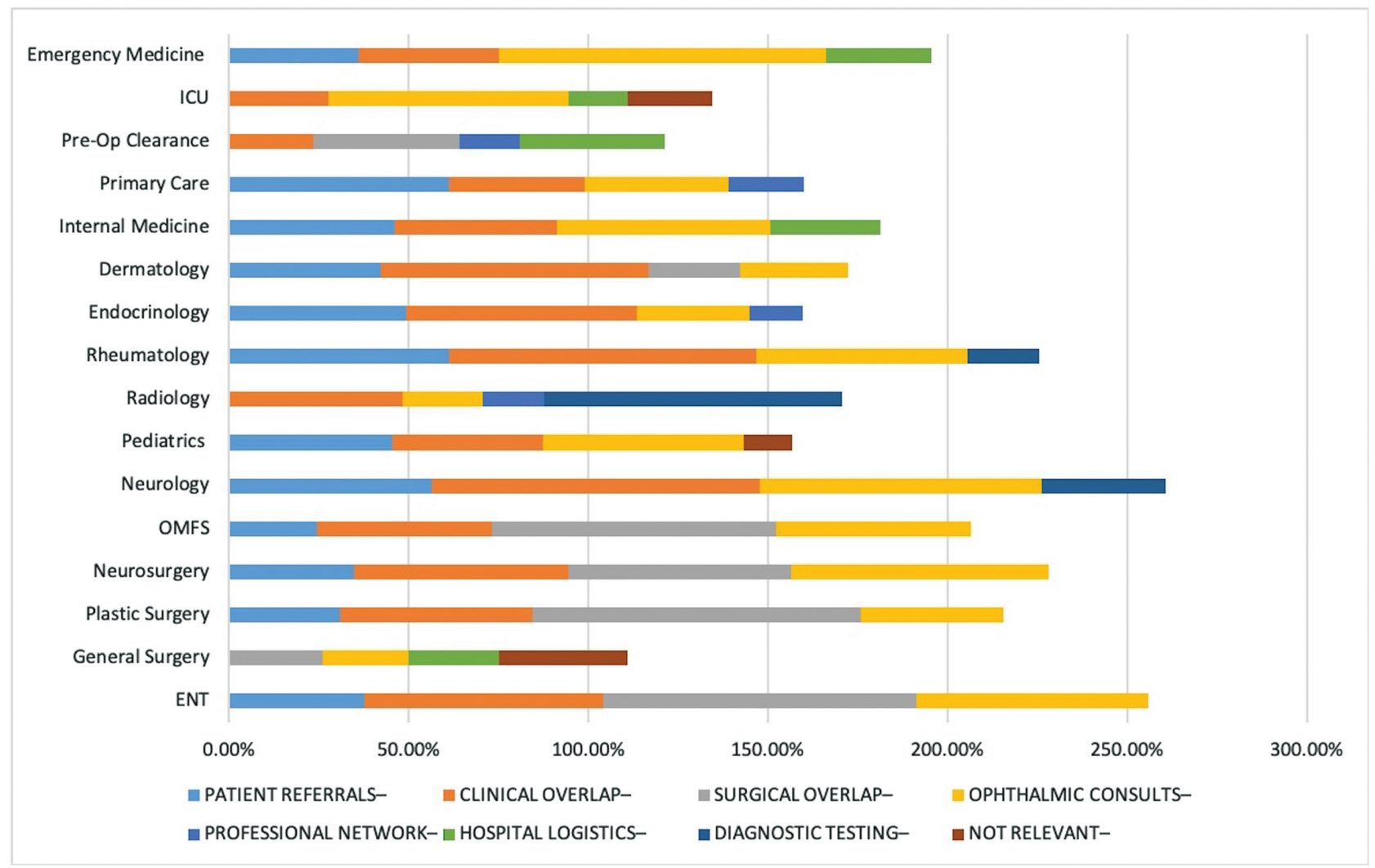

Fig. 2 Top 4 reasons for specialty inclusion. As up to three responses were allowed per specialty, total percentages in the $x$-axis can be more than $100 \%$. ENT, ears, nose, and throat; ICU, intensive care unit; OMFS, oral and maxillofacial surgery.

$5.22 / 10$, internship rating of 3 corresponded to an average of $5.36 / 10$, and internship ranking $\geq 4$ corresponded to an average of 5.27/10. When examining particular specialties, endocrinology and general surgery were ranked lower by survey takers who had poorer intern year experiences on initial analysis ( $p=0.02$ and $p=0.04$ ), but this was rendered insignificant on post hoc analysis. Mixed model analysis demonstrated that only urban location was a significant factor in higher specialty ratings ( $p=0.04,-$ Table 3$)$.

\section{Discussion}

As residency programs consider internship possibilities, trainee input into specialty relevance may be helpful. In this anonymous national survey, there were several specialtiesnotably ENT, radiology, and inpatient neurology-that trainees considered to be highly relevant but lacking in preresidency exposure. These findings are an opportunity to tailor the internship curriculum when possible to provide greater exposure to otherwise relatively neglected areas. The preference for neuroradiology, in particular, was almost unanimous. Ophthalmology residents often function as the first point of contact for a patient with a suspected intracranial or orbital lesion and vague ocular symptoms. A basic understanding of neuroradiologic diagnostic testing and interpretation is paramount to appropriate evaluation and referral. ${ }^{6}$

In contrast, many respondents noted extensive general surgery and ICU experience, but found these two specialties to be minimally relevant. Studies evaluating surgical apti- tude in ophthalmology residents measure a skillset that does not fully overlap with the skills gained by a general surgery experience, though presumably measures such as "flow of operation," "procedural knowledge," and "interaction with assistants/scrub nurse" are synonymous across surgical borders. $^{7}$ It is possible that this dichotomy is due to the relegation of many surgical interns to caring for pre- and postoperative patients on the floor. It is therefore unclear whether replacing general surgery experience with other, potentially more relevant surgical specialties, would change daily experience, though exposure to craniofacial pathology and anatomy may be greater.

Emergency medicine was most applicable to ophthalmology residents in terms of ophthalmology consults (91.01\%) and navigating hospital logistics (29.21\%). Surprisingly, emergency medicine was also considered less relevant compared with several other specialties, with a rating of only 5.6/10, despite high clinical exposure during consults. Ocular consults account for only $5 \%$ of all trauma-related visits to an urban emergency department (ED) and only 1.3 to $1.5 \%$ of all ED visits, which may explain why respondents felt that a general ED experience was of little clinical utility. ${ }^{8-10}$ Ophthalmology residents may benefit from establishing contacts in the ED, though only $10.11 \%$ listed professional network as an important element of ED training. While pediatric ophthalmology is a pivotal and substantial aspect of residency training, most respondents felt pediatrics overall was less important. Rheumatology and endocrinology, conversely, had significant clinical overlap with ophthalmology but minimal medical school experience. 


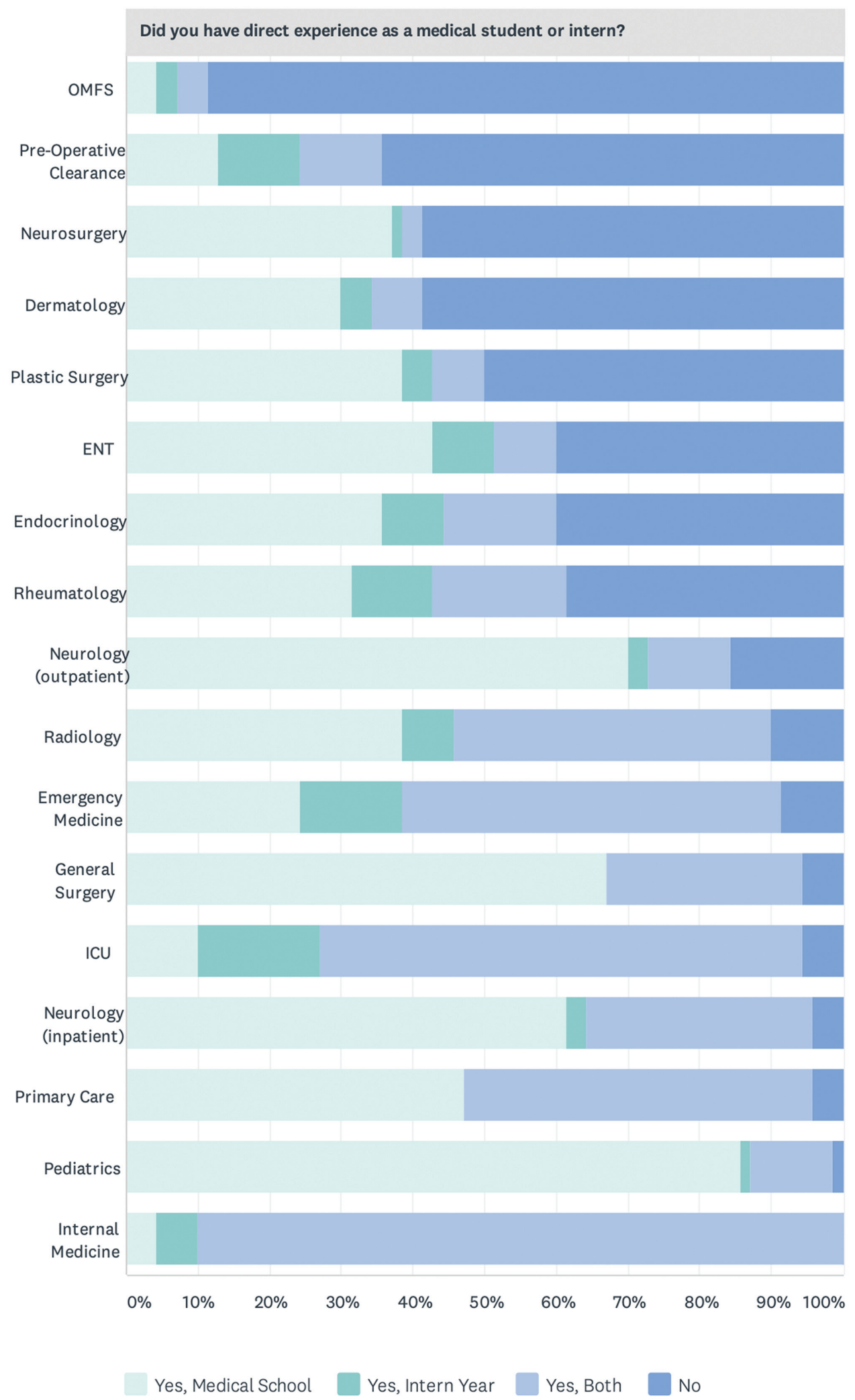

Fig. 3 Level of direct experience in each specialty. After rating the specialties' applicability to ophthalmology, participants were asked to state whether they had direct experience in the same specialty in medical school and/or internship. Specialties are organized from most to least for "no" experience whatsoever. ENT, ears, nose, and throat; ICU, intensive care unit; OMFS, oral and maxillofacial surgery.

Thus, incorporating rheumatology and endocrinology during internship might be of substantial educational utility.

It is unclear what accounted for the differences in specialty rating by urban versus nonurban respondents. Interestingly, residents from urban programs found primary care to be more valuable than their nonurban counterparts, in addition to other specialties, perhaps relating to a dearth of primary care specialists in urban centers across America. ${ }^{11-13}$ The 
Table 2 Extramural specialty applicability to ophthalmology by category

\begin{tabular}{|l|l|l|l|l|l|l|l|l|l|}
\hline Specialty (N) & $\begin{array}{l}\text { Urban } \\
(\mathbf{7 4})\end{array}$ & $\begin{array}{l}\text { Nonurban } \\
(\mathbf{2 0})\end{array}$ & $\begin{array}{l}\text { First } \\
\text { year } \\
(\mathbf{3 4})\end{array}$ & $\begin{array}{l}\text { Second } \\
\text { year } \\
(\mathbf{2 8})\end{array}$ & $\begin{array}{l}\text { Third year } \\
(\mathbf{2 3})\end{array}$ & $\begin{array}{l}\text { Fellow } \\
(\mathbf{9})\end{array}$ & $\begin{array}{l}\text { General } \\
\text { surgery } \\
(\mathbf{5})\end{array}$ & $\begin{array}{l}\text { Transitional } \\
(\mathbf{3 9})\end{array}$ & $\begin{array}{l}\text { IM } \\
(\mathbf{4 9})\end{array}$ \\
\hline ENT & 7.69 & 7.37 & 7.79 & 6.88 & 8.26 & 7.67 & 7.4 & 7.68 & 7.6 \\
\hline Radiology & 7.15 & 6.26 & 6.68 & 7.04 & 7.04 & 7.44 & 7.0 & 6.73 & 7.15 \\
\hline Neurology (inpatient) & 6.63 & 6.00 & 6.38 & 6.00 & 6.82 & 6.56 & 6.6 & 6.43 & 6.54 \\
\hline Plastic surgery & 6.28 & 6.79 & 6.21 & 6.57 & 6.30 & 6.00 & 7.2 & 6.42 & 6.28 \\
\hline Rheumatology & 6.34 & 5.89 & 5.91 & 6.04 & 6.52 & 7.00 & 5.2 & 6.19 & 6.4 \\
\hline OMFS & 6.17 & 4.89 & 5.97 & 5.54 & 6.61 & 5.33 & 5.8 & 5.70 & 6.06 \\
\hline Preoperative clearance & 5.85 & 5.79 & 5.35 & 5.79 & 6.26 & 6.11 & 5.8 & 5.57 & 6.04 \\
\hline Neurosurgery & 5.75 & 5.42 & 5.29 & 5.64 & 5.78 & 5.67 & 6.0 & 5.73 & 5.6 \\
\hline Emergency medicine & 5.81 & 4.84 & 5.10 & 5.43 & 6.17 & 5.44 & 5.4 & 5.63 & 5.60 \\
\hline Neurology (outpatient) & 5.35 & 4.75 & 5.15 & 5.04 & 5.30 & 5.78 & 4.4 & 5.37 & 5.19 \\
\hline Dermatology & 5.11 & 5.00 & 4.68 & 5.21 & 5.17 & 6.00 & 5.8 & 4.89 & 5.17 \\
\hline Endocrinology & 4.73 & 4.58 & 4.53 & 4.75 & 4.52 & 5.56 & 5.4 & 4.16 & 5.04 \\
\hline Internal medicine (inpatient) & 4.76 & 4.16 & 4.47 & 4.36 & 4.57 & 6.00 & 4.0 & 4.55 & 4.77 \\
\hline Pediatrics & 4.21 & 3.53 & 3.74 & 4.25 & 3.91 & 4.67 & 3.4 & 4.0 & 4.19 \\
\hline Primary care (outpatient) & 3.76 & 2.79 & 3.18 & 3.39 & 3.78 & 4.78 & 2.0 & 3.51 & 3.75 \\
\hline General surgery & 3.58 & 3.05 & 3.65 & 3.11 & 3.22 & 4.33 & 3.0 & 3.35 & 3.6 \\
\hline ICU & 3.07 & 1.89 & 2.59 & 2.60 & 3.26 & 3.00 & 2.4 & 2.81 & 2.88 \\
\hline Total average & 5.43 & 4.88 & 5.19 & 5.18 & 5.45 & 5.73 & 5.1 & 5.23 & 5.40 \\
\hline
\end{tabular}

Abbreviations: ENT, ears, nose, and throat; ICU, intensive care unit; IM, internal medicine; OMFS, oral and maxillofacial surgery. Note: Ratings by urban versus nonurban environment, year of training, and type of internship year (out of a 10-point scale).

greater desire for exposure to other specialties in more urban regions may be related to greater baseline exposure during residency, though of note, this survey did not ask respondents whether they attended urban or nonurban medical schools and internships.

Personal internship experience may have colored respondent ratings, with residents who had a subpar intern year experience $(\leq 2)$ ranking nearly all specialties more poorly, but this was not found to be statistically significant on post hoc and mixed model analysis. With an overall intern year experience of $4.03 / 5$, it is important to realize that most ophthalmology residents reported a high level of satisfaction with their internship. A previous survey conducted in 2018 indicated that most ophthalmology residents pick their internship based on geographic preference and perceived quality of life, which may influence internship ratings. ${ }^{14}$ Of note, this survey did not ask respondents how each specialty impacted daily quality of life, which may have colored responses. Our reported level of satisfaction, in conjunction with the findings by Logothetis et al, poses a positive challenge to the new internship curriculum. Of note, electives are commonplace in transitional programs, which ranked highest in resident satisfaction. It may be reasonable to offer personalized selections or electives, if possible.

This survey is limited by its lack of certain demographic information, such as gender, age, and additional degree experience. Nor did the survey ask participants if they had completed other research programs or residencies related to the specialties measured. The authors relied on the discretion of program coordinators/directors to forward the survey to their residents and fellows; as such, we do not know which programs were included or excluded. This may be a source of response bias, since we have an unknown response rate. Differences in perspective between years may also be a source of recall bias (e.g., earlier ophthalmology residents are closer to their internship experience than fellows). In total, $57.9 \%$ of all ophthalmology programs are medium size (4-6 residents/year), $32.7 \%$ are small ( $<4 /$ year), and $11.2 \%$ are large ( $>6 /$ year). In comparison, $75.1 \%$ of survey respondents were from medium-sized programs, $13.8 \%$ from small, and $10.6 \%$ from large. Finally, The severe acute respiratory syndrome coronavirus 2 (COVID-19) pandemic started sweeping across America after survey results had been gathered. Resident redeployment may influence current perceptions, and a repeat survey in the next year may be a useful tool. It is unknown whether this pandemic represents a once-in-a-lifetime event, in which case internship training may not need to be tailored to pandemic needs, or whether there will be an increased rate of pandemics requiring a deeper understanding of critical care.

\section{Conclusion}

The findings of this survey provide a framework to build a curricular plan based on current resident and fellow experience. Certain specialties were deemed highly valuable and also 
Table 3 Estimates of fixed effects on mixed model analysis

\begin{tabular}{|c|c|c|c|}
\hline Specialty & Estimate & $95 \% \mathrm{Cl}$ & Significance \\
\hline ENT & 2.13 & $1.62-2.64$ & 0.000 \\
\hline General surgery & -2.04 & -2.55 to -1.53 & 0.000 \\
\hline Plastic surgery & 0.83 & $0.32-1.34$ & 0.001 \\
\hline Neurosurgery & 0.06 & -0.45 to 0.57 & 0.807 \\
\hline OMFS & 0.45 & -0.06 to 0.96 & 0.087 \\
\hline Neurology (inpatient) & 0.90 & $0.39-1.42$ & 0.001 \\
\hline Neurology (outpatient) & -0.28 & -0.79 to 0.23 & 0.289 \\
\hline Pediatrics & -1.47 & -1.98 to -0.96 & 0.000 \\
\hline Radiology & 1.46 & $0.95-1.97$ & 0.000 \\
\hline Rheumatology & 0.71 & $0.20-1.22$ & 0.006 \\
\hline Endocrinology & -0.80 & -1.31 to -0.29 & 0.002 \\
\hline Dermatology & -0.40 & -0.92 to 0.11 & 0.121 \\
\hline Internal medicine & -0.88 & -1.39 to -0.37 & 0.001 \\
\hline Primary care & -1.95 & -2.46 to -1.44 & 0.000 \\
\hline Preoperative clearance & 0.29 & -0.22 to 0.80 & 0.271 \\
\hline ICU & -2.69 & -3.20 to -2.18 & 0.000 \\
\hline Emergency medicine & Reference & Reference & Reference \\
\hline \multicolumn{4}{|l|}{ Training year } \\
\hline PGY 2 & -0.55 & -1.39 to 0.30 & 0.200 \\
\hline PGY 3 & -0.46 & -1.32 to 0.40 & 0.292 \\
\hline PGY 4 & -0.14 & -1.01 to 0.73 & 0.756 \\
\hline Fellows & Reference & Reference & Reference \\
\hline \multicolumn{4}{|l|}{ Location } \\
\hline Urban & 0.57 & $0.01-1.13$ & 0.046 \\
\hline Nonurban & Reference & Reference & Reference \\
\hline \multicolumn{4}{|l|}{ Internship completed } \\
\hline Internal medicine & 0.28 & -0.87 to 1.44 & 0.626 \\
\hline Transitional & 0.09 & -1.07 to 1.26 & 0.873 \\
\hline General surgery & Reference & Reference & Reference \\
\hline \multicolumn{4}{|l|}{ Program size } \\
\hline$<4$ residents & -0.65 & -1.55 to 0.24 & 0.148 \\
\hline $4-6$ residents & -0.16 & -1.07 to 1.26 & 0.628 \\
\hline$>6$ residents & Reference & Reference & Reference \\
\hline \multicolumn{4}{|l|}{ Internship experience } \\
\hline Poor internship experience & 0.03 & -0.75 to 0.82 & 0.933 \\
\hline Average internship experience & 0.08 & -0.59 to 0.75 & 0.808 \\
\hline Good internship experience & Reference & Reference & Reference \\
\hline
\end{tabular}

Abbreviations: $\mathrm{Cl}$, confidence interval; ENT, ears, nose, and throat; ICU, intensive care unit; OMFS, oral and maxillofacial surgery; PGY, postgraduate year. Notes: Mixed model analysis output demonstrating the estimated extent to which certain factors impacted specialty relevance ratings. Several specialties were statistically significant when compared with emergency medicine as a reference group. For the other factors (year in training, location, type of internship completed, program size, and internship experience), being a fellow, nonurban program setting, general surgery internship, large program size, and good internship experience service as discrete comparative reference groups. Of these factors, only urban location remained statistically significant for differences in specialty ratings. 
demonstrated a lack of preresidency experience. This combination, notably ENT, neuroradiology, and neurology, provides the greatest opportunity for developing a desirable internship curriculum. Other specialties, such as ICU and general surgery, had robust preresidency experience with minimal overall relevance, suggesting that these subspecialties may be less useful to ophthalmology residency training. Replacing general surgery with ENT and OMFS, for example, may be a reasonable option to improve the diversity of experience and surgical overlap.

\section{Funding}

The Department of Ophthalmology at the Edward S. Harkness Eye Institute is supported by an unrestricted grant from Research to Prevent Blindness.

\section{Conflict of Interest \\ None declared.}

\section{References}

1 Oetting TA, Alfonso EC, Arnold A, et al. . Integrating the internship into ophthalmology residency programs: Association of University Professors of Ophthalmology American Academy of Ophthalmology white paper. Ophthalmology 2016;123(09):2037-2041

2 Osguthorpe JD. An otolaryngology residency: the evolution continues. Otolaryngol Head Neck 2004;131:795-796

3 Kovatch KJ, Harvey RS, Prince MEP, Thorne MC. National trends in otolaryngology intern curricula following Accreditation Council for Graduate Medical Education changes. Laryngoscope 2018;128 (08):1811-1816
4 Gong D, Winn BJ, Beal CJ, et al. . Gender differences in case volume among ophthalmology residents. JAMA Ophthalmol 2019;137 (09):1015-1020

52019 Ophthalmology Residency Match Summary Report. Association of University Professors of Ophthalmology 2019

6 Lee AG, Johnson MC, Policeni BA, Smoker WR. Imaging for neuroophthalmic and orbital disease - a review. Clin Exp Ophthalmol 2009;37(01):30-53

7 Cremers SL, Lora AN, Ferrufino-Ponce ZK. Global rating assessment of skills in intraocular surgery (GRASIS). Ophthalmology 2005;112(10):1655-1660

8 Wilson MR, Wooten F, Williams J. Frequency and characteristics of ocular trauma in an urban population. J Natl Med Assoc 1991;83 (08):697-702

9 Rizzuti AE, Vastardi M, Hajee M, Lazzaro DR. Scope of resident ophthalmology consultation service and patient follow-up rates at a level 1 trauma center in Brooklyn, New York. Clin Ophthalmol 2013;7:643-647

10 Heiferman MJ, Khanna S, Gu D, Agron S, Eichinger SE, Bryar PJ. Emergency department ophthalmology consultations in a tertiary care hospital. J Acad Ophthalmol 2019;11(01):e25-e29

11 Devi S. New York moves to tackle shortage of primary-care doctors. Lancet 2008;371(9615):801-802

12 Haggerty JL, Roberge D, Pineault R, Larouche D, Touati N. Features of primary healthcare clinics associated with patients' utilization of emergency rooms: urban-rural differences. Healthc Policy 2007;3(02):72-85

13 Kroneman M, Verheij R, Tacken M, van der Zee J. Urban-rural health differences: primary care data and self reported data render different results. Health Place 2010;16(05):893-902

14 Logothetis HD, Pyatetsky D, Baqai JA, Volpe NJ. Ophthalmology residents' internship selection and initial trainee confidence: an observational study. J Acad Ophthalmol 2018;10(01):e72-e78 